\title{
High activity redox catalysts synthesized by chemical vapor impregnation
}

\begin{abstract}
The use of precious metals in heterogeneous catalysis relies on the preparation of small nanoparticles that are stable under reaction conditions. To date, most conventional routes used to prepare noble metal nanoparticles have drawbacks related to surface contamination, particle agglomeration, and reproducibility restraints. We have prepared titania-supported palladium (Pd) and platinum (Pt) catalysts using a simplified vapor deposition technique termed chemical vapor impregnation (CVI) that can be performed in any standard chemical laboratory. These materials, composed of nanoparticles typically below $3 \mathrm{~nm}$ in size, show remarkable activity under mild conditions for oxidation and hydrogenation reactions of industrial importance. We demonstrate the preparation of bimetallic Pdï Pt homogeneous alloy nanoparticles by this new CVI method, which show synergistic effects in toluene oxidation. The versatility of our CVI methodology to be able to tailor the composition and morphology of supported nanoparticles in an easily accessible and scalable manner is further demonstrated by the synthesis of Pdshellï Aucore nanoparticles using CVI deposition of Pd onto preformed $\mathrm{Au}$ nanoparticles supported on titania (prepared by sol immobilization) in addition to the presence of monometallic $\mathrm{Au}$ and Pd nanoparticles.
\end{abstract}

Keyword: Bimetallic nanoparticle; Catalysis; Core-shell structures; Gold; Hydrogenation; Nanoalloy; Oxidation; Palladium; Platinum 